Received 10272019

Accepted 12152019

Published $12 \quad 30 \quad 2019$

CONTENT OF PHOSPHORUS AND

ARSENIC, AND OF OTHER

CONTAMINATING METALS OF THE

SURFACE WATERS OF THE COATA

RIVER, AN AFFLUENT OF LAKE

TITICACA, PERU

\section{DETERMINACIÓN DEL CONTENIDO DE FÓSFORO Y ARSÉNICO, Y DE OTROS METALES CONTAMINANTES DE LAS AGUAS SUPERFICIALES DEL RÍO COATA, AFLUENTE DEL LAGO TITICACA, PERÚ}

Full original article

Vol. 36, No.5, pp. 223-228, Nov./Dic.2019

Revista Boliviana de Química

36(5), 223-228, Nov./Dec. 2019

Bolivian Journal of Chemistry

DOI : $10.34098 / 2078-3949.36 .5 .4$

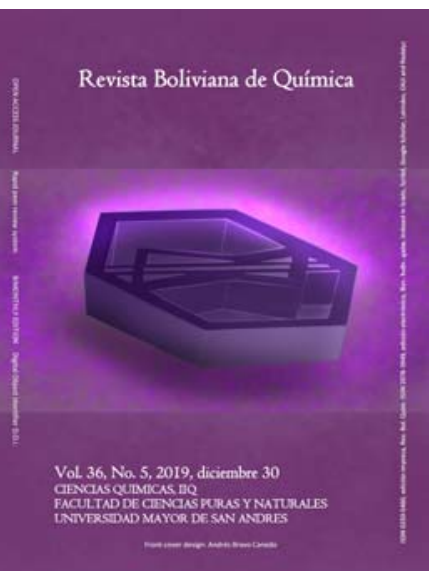

Peer-reviewed

Germán Belizario Quispe ${ }^{1,4}$; Javier Capacoila Coila; Edilberto Huaquisto Ramos ${ }^{1}$; Dalmiro A. Cornejo Olarte2; Heber N. Chui Betancur ${ }^{3,4, *}$

${ }^{1}$ Escuela Profesional de Ingeniería Agrícola, Universidad Nacional del Altiplano de Puno, Av. Floral No 1153 , Puno, Perú. belge29@hotmail.com

${ }^{2}$ Escuela Profesional de Ingeniería Metalúrgica, Universidad Nacional del Altiplano de Puno, Av. Floral № 1153, Puno, Perú

${ }^{3}$ Instituto de Investigación de la Escuela de Posgrado, Universidad Nacional del Altiplano de Puno, Av. Floral No 1153, Puno, Perú

${ }^{4}$ Instituto de Investigación de Recursos Hídricos, Universidad Privada San Carlos SAC, Conde de Lemos 128, Puno, Perú

Keywords: Superficial waters, Water quality, Pollution, Evaluation, Phosphorus, Arsenic, Coata river, Perú

Palabras clave: Aguas superficiales, Calidad del agua, Contaminación, Fósforo, Arsénico, Río Coata.

\title{
ABSTRACT
}

The contents of phosphorus, arsenic, aluminum, iron and manganese in the surface waters of the Coata River, tributary of Lake Titicaca, department of Puno, Peru, were measured. The measurements were made at two times of the year: sewer and avenue, and were applied at five strategic points on the Coata River, from the Independence Bridge in the city of Juliaca to its mouth on Lake Titicaca. The inductive coupling plasma (ICP) technique consisting of an ionization source and an optical emission spectrophotometer (OES) was used to determine the 
REVISTA BOLIVIANA DE QUÍMICA

ISSN 0250-5460 Rev. Bol. Quim. Paper edition

ISSN 2078-3949 Rev, boliv, quim. Electronic edition

Germán Belizario Quispe et al. RBQ Vol. 36, No.5, pp. 223-228, 2019

elements. The measurements of the concentrations were made by atomic emission spectrometry. The maximum concentrations determined were: aluminum 1,043 mg / L, iron $0.856 \mathrm{mg} / \mathrm{L}$, manganese $0.460 \mathrm{mg}$ / L, arsenic 0.029 $\mathrm{mg} / \mathrm{L}$ and phosphorus 10,287 mg / L, indices that exceed the permissible limits established in the Environmental Quality Standards of Ministry of Environment of Peru. The thus detected contamination of river waters has its origin in the uncontrolled and untreated discharge of wastewater and solid waste from the city of Juliaca, Puno, Peru.

\section{Corresponding author: heberchui@gmail.com}

\section{RESUMEN}

Se midió los contenidos de fósforo, arsénico, aluminio, hierro y manganeso en las aguas superficiales del río Coata, afluente del lago Titicaca, departamento de Puno, Perú. Las mediciones fueron hechas en dos épocas del año: estiaje y avenida, y realizadas en cinco puntos estratégicos del rio Coata, desde el puente Independencia de la ciudad de Juliaca hasta su desembocadura en el lago Titicaca. Para la determinación de los elementos se empleó la técnica de plasma de acoplamiento inductivo (ICP) que consta de una fuente de ionización, y de un espectrofotómetro de emisión óptica (OES). Las mediciónes de las concentraciónes fueron realizadas por espectrometría de emisión atómica. Las concentraciones máximas determinadas fueron: aluminio $1.043 \mathrm{mg} / \mathrm{L}$, hierro $0.856 \mathrm{mg} / \mathrm{L}$, manganeso $0.460 \mathrm{mg} / \mathrm{L}$, arsénico $0.029 \mathrm{mg} / \mathrm{L}$ y fósforo $10.287 \mathrm{mg} / \mathrm{L}$, índices que exceden los límites permisibles establecidos en los Estándares de Calidad Ambiental del Ministerio del Ambiente de Perú. La así detectada contaminación de las aguas fluviales tiene su origen en la descarga incontrolada y sin tratamiento de las aguas residuales y residuos sólidos de la ciudad de Juliaca, Puno, Perú.

\section{INTRODUCCIÓN}

Los efectos sobre la ecología y en consecuencia sobre la vida, de la presencia de elementos químicos de origen natural o antropogénico, que sobrepasen la norma en contenidos, definen a dichos elementos como contaminantes y desvirtúan el carácter de inocuidad del agua, necesario para su consumo humano y para las actividades relacionadas al mismo [1]. Dichos efectos son de naturaleza física, química y biológica, y provocan una degradación de la calidad original de los ecosistemas y de los suelos agrícolas [2-5]. La alteración de suelos, recursos hídricos y aire por metales pesados y metaloides proyecta una tendencia de la problemática más severa que compromete la seguridad alimentaria y salud pública a nivel local y mundial [6,7].

La contaminación fluvial tiene como causales las actividades extractivas de oro responsables de la contaminación por arsénico (As) [8,9], y la contaminación por aguas residuales citadinas [10], responsables de una variedad de elementos nocivos que por bioacumulación en los tejidos de los seres vivos acuáticos pueden provocar desórdenes fisiológicos [11]. Por lo tanto, la calidad del agua es un factor determinante para la salud de humanos fauna y flora.

Los ecosistemas acuáticos cercanos a las ciudades muy pobladas presentan concentraciones relativamente elevadas de fósforo [13], con la consecuente alteración de los parámetros acuíferos y del orden ecológico [14]. La vida acuática depende, entre otras cosas, de la cantidad de nitrógeno $(\mathrm{N})$ y fósforo $(\mathrm{P})$ que está disponible en el ecosistema acuático [13]. Por lo general, en los sistemas acuáticos existe una mayor proporción de nitrógeno que de fósforo y las especies de estos ecosistemas han evolucionado para habitar en estas condiciones [15,16]. Pero, si esta proporción se altera, los organismos reducen su capacidad de crecimiento y mantenimiento de las funciones vitales. Sin embargo, el vertido continuo de detergentes, fertilizantes, pesticidas, aguas residuales urbanas [17] y desechos industriales [18] a las cuencas fluviales añaden cantidades desorbitantes de fósforo [19], lo que disminuye proporcionalmente la cantidad de nitrógeno. Así, el fósforo pasa de ser un nutriente deseable a ser un contaminante de sistemas acuáticos cercanos a ciudades densamente pobladas. La presencia excesiva de nitrógeno y de fósforo favorece un proceso de eutrofización del agua [16]. Este proceso resulta en un crecimiento descontrolado de algunas especies acuáticas en la superficie que terminan por impedir el paso de la luz [20], por lo que las algas del fondo no pueden realizar la fotosíntesis y el agua acaba perdiendo casi todo el oxígeno [4,21,22].

El propósito de este trabajo es evaluar la presencia de arsénico y fósforo en las aguas superficiales en la parte baja de la cuenca de río Coata, desde el puente Independencia de la ciudad de Juliaca hasta su desembocadura en el Titicaca. 


\section{Instrumentos}

Se empleó la cadena de custodia, en la que se hizo uso de envases de polietileno (ziploc) lavados con la misma agua y previamente etiquetados, a su vez preservados en bolsas plásticas negras y mantenidos en refrigeración hasta su ingreso a los laboratorios de Ensayo y Control de Calidad de la Universidad Católica Santa María - Arequipa con sus registros en la cadena de custodia, y su análisis de espectrometría de emisión atómica respectivos, según el método EPA 200.7. Para la determinación de elementos traza en las muestras del río Coata, se utilizó el plasma de acoplamiento inductivo-ICP con una fuente de ionización junto a un espectrofotómetro de emisión óptico-OES. Estos resultados se compararon con los límites permisibles establecidos por los Estándares de Calidad Ambiental de agua del Ministerio del Ambiente. Se analizó el punto más crítico, en función a la conductividad eléctrica tomadas en el agua in situ.

\section{Materiales y métodos}

Las muestras fueron colectadas en la cuenca baja del rio Coata, desde la ciudad de Juliaca, hasta su desembocadura en el lago Titicaca, en dos periodos climáticos: avenida (marzo) y estiaje (junio) en el año 2017, en cinco puntos estratégicos que fueron georreferenciados (Tabla 1).

Tabla 1. Datos de localización de las muestras.

\begin{tabular}{llll}
\hline Muestra & (WGS84) Este & (WGS84) Norte & Altitud \\
\hline P1 - Puente Independencia & 381478.13 & 8290421.62 & $3833 \mathrm{msnm}$ \\
P2 - Desembocadura del río Torococha & 385267.51 & 8285422.64 & $3830 \mathrm{msnm}$ \\
P3 - Mayoesquina & 390758.44 & 8280058.72 & $3828 \mathrm{msnm}$ \\
P4 - Puente Grande & 397450.95 & 8278882.54 & $3825 \mathrm{msnm}$ \\
P5 - Desembocadura del río Coata & 402797.59 & 8275181.69 & $3821 \mathrm{msnm}$ \\
\hline
\end{tabular}

Se colectó muestras de agua de río por duplicado in situ, tomando en cuenta los parámetros químicos y físicos a saber: CND, pH y temperatura en cada uno de los cinco puntos de muestreo (Tabla 1), en las dos épocas, usándose un recipiente graduado y recipientes de polietileno de $500 \mathrm{~mL}$.

\section{RESULTADOS Y DISCUSIÓN}

Se determinó la presencia de aluminio, hierro y manganeso con concentraciones por encima de los límites permisibles establecidos por los ECAs del Minam-Perú, índices preocupantes por la alteración de los sistemas acuáticos [23].

Tabla 2. Concentración en $\mathrm{mg} / \mathrm{L}$ de elementos químicos: aluminio, hierro y manganeso en aguas superficiales del rio Coata y normas ECA- Minam en $\mathrm{mg} / \mathrm{L}$.

\begin{tabular}{lllllll}
\hline \multirow{2}{*}{$\begin{array}{l}\text { Punto de } \\
\text { muestreo }\end{array}$} & \multicolumn{3}{c}{ Época de avenida } & \multicolumn{3}{c}{ Época de estiaje } \\
\cline { 2 - 7 } & Aluminio & Hierro & Manganeso & Aluminio & Hierro & Manganeso \\
\hline P-01 & 0.718 & 0.594 & 0.042 & 0.000 & 0.000 & 0.000 \\
P-02 & 0.279 & 0.230 & 0.106 & 0.142 & 0.184 & 0.460 \\
P-03 & 0.000 & 0.856 & 0.086 & 0.036 & 0.000 & 0.000 \\
P-04 & 0.000 & 0.689 & 0.057 & 0.000 & 0.000 & 0.000 \\
P-05 & 1.043 & 0.821 & 0.080 & 0.017 & 0.000 & 0.000 \\
Normas ECA & 0.900 & 0.300 & 0.400 & 0.900 & 0.300 & 0.400 \\
\hline
\end{tabular}

Downloadable from: Revista Boliviana 225 de Química. Volumen 36 N5. Año 2019

http://www.bolivianchemistryjournal.org, http://www.scribd.com/bolivianjournalofchemistry 


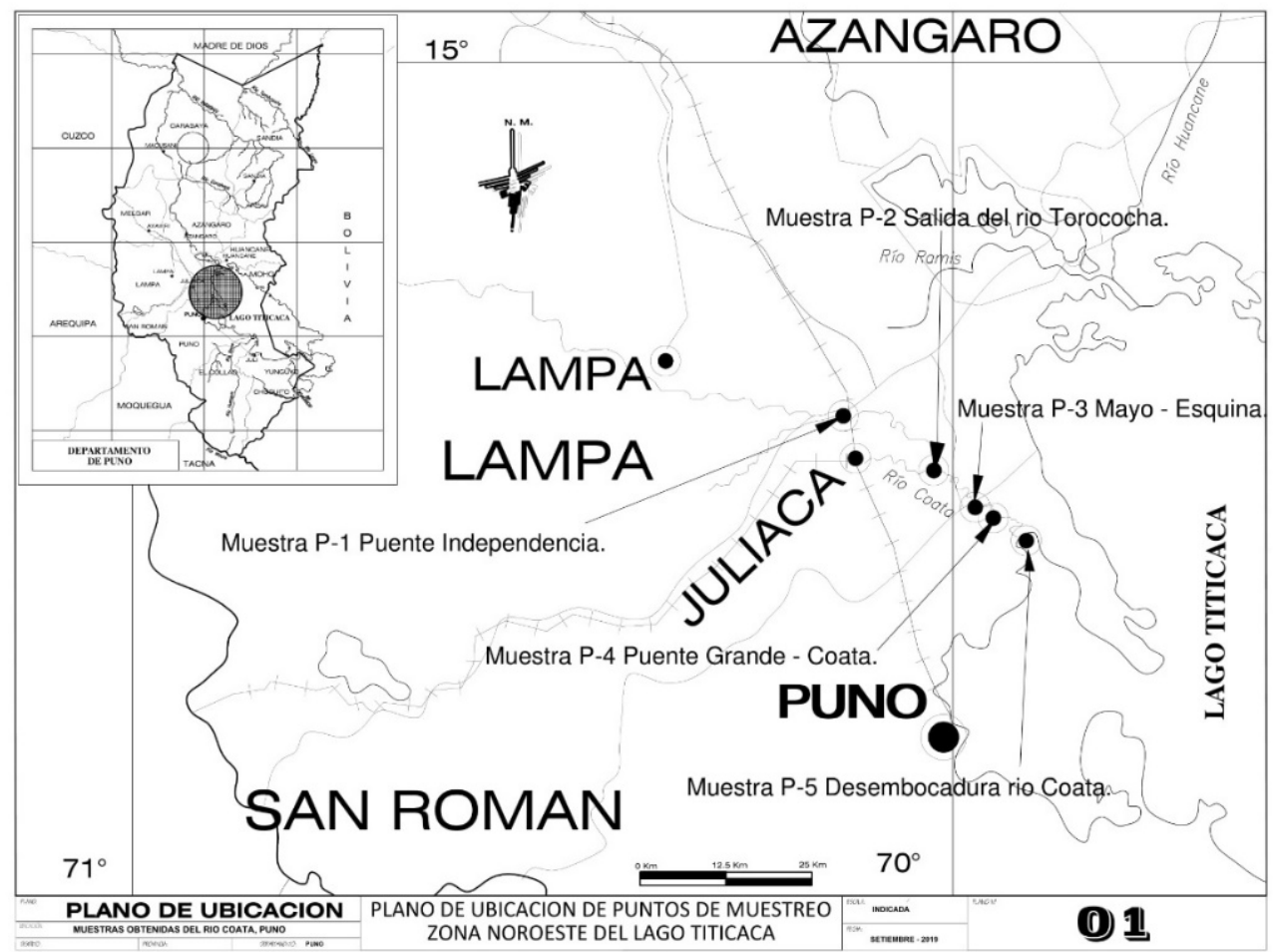

Figura 1. Ubicación de puntos de muestreo del rio Coata.

La mayoría de los elementos estudiados exceden los límites permisibles en agua según los Estándares de Calidad Ambiental-ECA establecido por el Ministerio del Ambiente de Perú. En la época de avenida el aluminio en el punto muestreo P-5 y el hierro en los puntos de muestreo P-01, P-03, P-04 y P-05 superan los mencionados límites, mientras que en la época de estiaje, el manganeso en el punto de muestreo P-2 supera dichos límites (Tabla 2). Las actividades humanas realizadas en la cuenca baja del río Coata, generan contaminantes para los cuerpos receptores como ser aguas superficiales y fauna y flora subacuática y rivereña, que ven así su hábitat alterado con la alteración del ecosistema consecuente [1,28] así como la alteración de los suelos de cultivo aledaños [2-5] con la subsecuente amenaza a la seguridad alimentaria y salud pública [6,7,9,].

Tabla 3. Concentración en $\mathrm{mg} / \mathrm{L}$ de Arsénico, Boro y Fósforo en las aguas superficiales de rio Coata y normas ECAMinam en $\mathrm{mg} / \mathrm{L}$.

\begin{tabular}{llllllll}
\hline \multirow{2}{*}{$\begin{array}{l}\text { Punto de } \\
\text { muestreo }\end{array}$} & Unidad & \multicolumn{3}{c}{ Época de avenida } & \multicolumn{3}{c}{ Época de estiaje } \\
\cline { 3 - 8 } & & Arsénico & Boro & Fósforo & $\begin{array}{l}\text { Arsénic } \\
\text { o }\end{array}$ & Boro & Fósfor \\
& & & & & & 0 \\
\hline P-01 & $\mathrm{mg} / \mathrm{L}$ & 0.000 & 0.054 & 0.035 & 0.027 & 0.713 & 0.660 \\
P-02 & mg/L & 0.000 & 0.105 & 1.254 & 0.022 & 0.816 & 10.287 \\
P-03 & mg/L & 0.000 & 0.047 & 0.141 & 0.028 & 0.775 & 0.345 \\
P-04 & mg/L & 0.000 & 0.051 & 0.000 & 0.000 & 0.790 & 0.328 \\
P-05 & mg/L & 0.013 & 0.062 & 0.065 & 0.029 & 0.777 & 0.333 \\
Normas ECA & mg/L & 0.001 & 2.400 & 0.100 & 0.001 & 2.400 & 0.100 \\
\hline
\end{tabular}


En la época de avenida el arsénico en el Punto P-05 (desembocadura del rio Coata) y el fósforo en los puntos de muestreo P-02 y P-03 superan los límites permisibles, mientras que en la época de estiaje el arsénico en los puntos de muestreo P-01, P-02, P-03 y P-05 y el fósforo en todos los puntos de muestreo superan los niveles permitidos de los ECA-Minam (Tabla 3). La excesiva concentración de fósforo que supera los límites permisibles $(0.100 \mathrm{mg} / \mathrm{L})$ en los ecosistemas acuáticos como es el caso del río Coata [13,14]. El incremento de la concentración de fósforo disminuye la concentración proporcional de nitrógeno [19], con la consecuente alteración de la habitabilidad del ambiente, reduciendo significativamente la capacidad de crecimiento y mantenimiento de las funciones vitales de los seres acuáticos [15-18]. La presencia elevada de fósforo favorece el proceso de eutrofización del agua [16], este proceso involucra un crecimiento descontrolado de algunas especies acuáticas en la superficie que terminan por impedir el paso de la luz [20], por lo que las algas del fondo no pueden realizar la fotosíntesis y el agua acaba perdiendo casi todo su oxígeno $[4,21,22]$.

Tabla 4. Pruebas in situ: temperatura $\left({ }^{\circ} \mathrm{C}\right)$, conductividad eléctrica $(\mu \mathrm{S} / \mathrm{cm})$ y $\mathrm{pH}$ en las aguas superficiales en las épocas de avenida (marzo) y estiaje (junio), río Coata - 2017.

\begin{tabular}{lllllll}
\hline \multirow{2}{*}{$\begin{array}{c}\text { Punto de } \\
\text { muestreo }\end{array}$} & \multicolumn{3}{c}{ Época de avenida } & \multicolumn{4}{c}{ Época de estiaje } \\
\cline { 2 - 7 } & Temperatura & $\begin{array}{l}\text { Conductividad } \\
\text { eléctrica }\end{array}$ & $\mathrm{pH}$ & Temperatura & $\begin{array}{l}\text { Conductividad } \\
\text { eléctrica }\end{array}$ & $\mathrm{pH}$ \\
\hline P-01 & 13 & 164 & 7.31 & 10 & 870 & 9.80 \\
P-02 & 16.5 & 380 & 7.60 & 13 & 1680 & 8.40 \\
P-03 & 15 & 170 & 8.30 & 14.5 & 460 & 8.50 \\
P-04 & 15.3 & 175 & 8.80 & 14 & 915 & 8.25 \\
P-05 & 13.7 & 160 & 8.30 & 14 & 455 & 9.20 \\
Máximo & 16.5 & 380 & 8.80 & 14.5 & 1680 & 9.80 \\
Mínimo & 13 & 160 & 7.31 & 13 & 455 & 8.25 \\
\hline
\end{tabular}

La temperatura del agua fluctuó entre $13^{\circ} \mathrm{C}$ y $16^{\circ} \mathrm{C}$, e influyó en la velocidad de los procesos fisiológicos de organismos, tales como la respiración microbiana que es responsable de gran parte de la auto-purificación en los cuerpos de agua.

En la época de avenida los valores de la conductividad eléctrica fueron desde $160 \mu \mathrm{S} / \mathrm{cm}$ (en la desembocadura del río Coata), hasta $380 \mu \mathrm{S} / \mathrm{cm}$ en la (desembocadura del río Torococha, (tomado como referencia de río con efectos antropogénicos); mientras que en la época de estiaje, se tuvo un resultado mínimo de $455 \mu \mathrm{S} / \mathrm{cm}$ y un máximo de $1680 \mu \mathrm{S} / \mathrm{cm}$ en los mismos puntos de muestreo antes indicados (Tabla 4). Según la tabla 4, éste último valor supera el límite establecido por la ECA para categoría I (A1) $(1500 \mu \mathrm{S} / \mathrm{cm})$ que es básicamente debido al ingreso de las aguas residuales de la ciudad de Juliaca por el río Torococha en el P-2 en época de estiaje y en la época de avenida es más diluida.

El aumento de la conductividad es debido a la presencia de sales, iones disueltos y materia orgánica disuelta, asimismo, la conductividad puede ser influenciada por el $\mathrm{pH}$, éstas son las condiciones adecuadas para generar eutrofización en las partes bajas de la cuenca y el lago Titicaca. La conductividad de la mayoría de las aguas dulces es de 10 a $1000 \mu \mathrm{S} / \mathrm{cm}$, pero puede exceder los $1000 \mu \mathrm{S} / \mathrm{cm}$, especialmente en aguas contaminadas, y más cuando reciben grandes cantidades de afluentes de aguas residuales [24], siendo así un indicador de contaminación y vulnerabilidad. En lo referido al pH, el mínimo es de 7.3 (P-1) y el máximo de 8.8 (P-4) en las épocas de avenida, mientras en estiaje el valor mínimo es de 8.25 (P-4) y el máximo de 9.8 (P-1), estos valores hacen que las aguas sean más básicas o alcalinas. Para el agua potable, la directriz de la OMS establece que el pH sea de 6.5 a 8.5. Sin embargo, el $\mathrm{pH}$ en aguas naturales está entre 6.0 y 8.5, aunque valores más bajos a 6.0 pueden ocurrir en aguas diluidas con alto contenido de materia orgánica, y valores más altos a 8.5 en aguas eutróficas, en aguas subterráneas $\mathrm{y}$ en lagos salados $[25,26]$.

\section{CONCLUSIONES}


La concentración de metales y fósforo en dos épocas de muestreo de las aguas superficiales en la parte baja de la cuenca del río Coata fue determinada: aluminio $1.043 \mathrm{mg} / \mathrm{L}$ y $0.142 \mathrm{mg} / \mathrm{L}$, hierro $0.856 \mathrm{mg} / \mathrm{L}$ y $0.184 \mathrm{mg} / \mathrm{L}$, manganeso $0.106 \mathrm{mg} / \mathrm{L}$ y $0.460 \mathrm{mg} / \mathrm{L}$. Las concentraciones de arsénico $0.029 \mathrm{mg} / \mathrm{L}$ y fósforo $10.287 \mathrm{mg} / \mathrm{L}$ en época de estiaje superan los niveles permisibles de los ECA Minam. La conductividad eléctrica fue de $1680 \mu \mathrm{S} / \mathrm{cm}$. Las causas de la contaminación fueron antropogénicas por el vertido de aguas residuales de la ciudad de Juliaca.

\section{REFERENCIAS}

1. Gallardo-Martínez, D., Bruguera-Amarán, N., Díaz-Duque, J.A., Cabrera-Díaz, I. 2015, Impacto provocado por la minería en la zona de Santa Lucía: evaluación físico--química, Minería y Geol., 31 (4), 100-120.

2. Alahabadi, A., Malvandi, H. 2018, Contamination and ecological risk assessment of heavy metals and metalloids in surface sediments of the Tajan River, Iran, Mar. Pollut. Bull., 133 (10), 741-749.

3. Mohammad, M., Lokman, M., Islam, S., Rahman, Z. 2016, Preliminary assessment of heavy metals in water and sediment of Karnaphuli River, Bangladesh, Environ. Nanotechnology, Monit. Manag., 5, 27-35.

4. Saiful, I., Belal, H., Matin, A., Shafiqul Islam, S. 2018, Assessment of heavy metal pollution, distribution and source apportionment in the sediment from Feni River estuary, Bangladesh, Chemosphere, 202, 25-32.

5. Kang, M., Tian, Y., Peng, S., Wang, M. 2019, Effect of dissolved oxygen and nutrient levels on heavy metal contents and fractions in river surface sediments, Sci. Total Environ., 648, 861-870.

6. Duodu, G.O., Goonetilleke, A., Ayoko, G.A. 2016Comparison of pollution indices for the assessment of heavy metal in Brisbane River sediment, Environ. Pollut., 219, 1077-1091.

7. Zhang, S., Zhang, J., Jing, X., Wang, Y., Wang, Y., Yue, T. 2018 Water saving efficiency and reliability of rainwater harvesting systems in the context of climate change, J. Clean. Prod., 196, 1341-1355.

8. Barral-Fraga, L., Martiñá-Prieto, Barral, D.T., Morin, S., Guasch, H. 2018, Mutual interaction between arsenic and biofilm in a mining impacted river, Sci. Total Environ., 636, 985-998.

9. Yañez, L.M., Alfaro, J.A., Avila Carreras, N.M.E., Bovi Mitre, G. 2019, Arsenic accumulation in lettuce (Lactuca sativa L.) and broad bean (Vicia faba L.) crops and its potential risk for human consumption, Heliyon, 5 (1) e01152 d.o.i.: 10.1016/j.heliyon.2019.e01152.

10. Ariffin, M., Sulaiman, S.N.M. 2015, Regulating Sewage Pollution of Malaysian Rivers and its Challenges, Procedia Environ. Sci., 30, 168-173.

11. Perera, P.C.T., Sundarabarathy, T.V., Sivananthawerl, T., Kodithuwakku, S.P., Edirisinghe, U. 2016, Arsenic and Cadmium Contamination in Water, Sediments and Fish is a Consequence of Paddy Cultivation: Evidence of River Pollution in Sri Lanka, Achiev. Life Sci., 10 (2), 144-160.

12. Zhao, Y. Xia, X.H., Yang, Z.F.M., Wang, F. 2012, Assessment of water quality in Baiyangdian Lake using multivariate statistical techniques, Procedia Environ. Sci., 13, 1213-1226.

13. Westphal, K., Graeber, D., Musolff, A., Fang, Y., Jawitz, J.W., Borchardt, D. 2019, Multi-decadal trajectories of phosphorus loading, export, and instream retention along a catchment gradient, Sci. Total Environ., 667, 769-779.

14. Moronta-Riera, J.L., Riverón-Zaldivar, A.B. 2016, Evaluación de la calidad físico-química de las aguas y sedimentos en la costa oriental del lago de Maracaibo, Minería y Geol., 32 (2), 102-111.

15. Koch, S., Kahle, P., Lennartz, B. 2018, Spatio-temporal analysis of phosphorus concentrations in a North-Eastern German lowland watershed, J. Hydrol. Reg. Stud., 15, 203-216.

16. Dambeniece-Migliniece, L., Veinbergs, A., Lagzdins, A. 2018, The impacts of agricultural land use on nitrogen and phosphorus loads in the Mellupite catchment, Energy Procedia, 147, 189-194.

17. Mockler, E.M., Deakin, J., Archbold, M., Gill, L., Daly, D., Bruen, M. 2017, Sources of nitrogen and phosphorus emissions to Irish rivers and coastal waters: Estimates from a nutrient load apportionment framework, Sci. Total Environ., 601-602, 326-339. doi: 10.1016/j.scitotenv.2017.05.186.

18. Valencia-Quintana, R., Sánchez-Aalarcón, J., Ortiz-Ortiz, E., Gómez-Olivares, J.L. 2007, La contaminación de los ríos, otro punto de vista, Rev. Cienc. y Tecnol. la UACJ, V, (1), 35-49.

19. Cassidy, R., Thomas, I.A., Higgins, A., Bailey, J.S., Jordan, P. A carrying capacity framework for soil phosphorus and hydrological sensitivity from farm to catchment scales, Sci. Total Environ., 687, 277-286.

20. Yan, Z. 2016, Phosphorus accumulates faster than nitrogen globally in freshwater ecosystems under anthropogenic impacts,” Ecol. Lett., 19 (10), 1237-1246.

21. Liao, J., Chen, J., Ru, X., Chen, J., Wu, H.,Wei, C. 2017, Heavy metals in river surface sediments affected with multiple pollution sources, South China: Distribution, enrichment and source apportionment, J. Geochemical Explor., 176, 9-19.

22. Zhang, G. 2017, Heavy metal fractions and ecological risk assessment in sediments from urban, rural and reclamation-affected rivers of the Pearl River Estuary, China, Chemosphere, 184, 278-288.

23. Marcela, L., Betancur, A. / Karina, Mazo, I.M., José, A., Mendoza, S. 2005, Fitorremediación: la alternativa para absorber metales pesados de los biosólidos, Rev. Lasallista Investig., 2 (1), 57-60.

24. Nhut, P., Chapman, L., Tight, M. 2019, Resilient transport systems to reduce urban vulnerability to fl oods in emerging-coastal cities: A case study of Ho Chi Minh City, Vietnam, Travel Behav. Soc., 15, (9), 28-43.

25. WHO, Environmental Health Criteria 101: Methylmercury, Genova, 1993.

26. Chapman, D. Water Quality Assessments - A Guide to Use of Biota, Sediments and Water in Environmental Monitoring, E\&FN Spon, an imprint of Chapman \& Hall, $2^{\text {nd }}$ ed, 1992. UNESCO/WHO/UNEP, Cambridge, 288-294.

27. Gutiérrez, F, Desiderio; García, Segmentación ecológica en destinos turísticos, Estudios turísticos, 149, 23-38.

Downloadable from: Revista Boliviana 228 de Química. Volumen 36 №5. Año 2019

http://www.bolivianchemistryjournal.org, http://www.scribd.com/bolivianjournalofchemistry 